\begin{tabular}{|c|c|}
\hline Title & Near-IR vibrational dynamics of periodic gold single and pair nanocuboids \\
\hline Author(s) & W ang, Li; Nishijima, Y oshiaki; U eno, Kosei; Misawa, Hiroaki; Tamai, Naoto \\
\hline Citation & $\begin{array}{l}\text { A pplied Phy sics Letters, 95(5), } 053116 \\
\text { https://doi.org/10.1063/1.3202403 }\end{array}$ \\
\hline Issue Date & 2009-08-03 \\
\hline Doc URL & http:/hdl.handle.net/2115/39067 \\
\hline Rights & $\begin{array}{l}\text { Copyright } \odot 2009 \text { A merican Institute of Physics. This article may be downloaded for personal use only. A ny other use } \\
\text { requires prior permission of the author and the A merican Institute of Phy sics. }\end{array}$ \\
\hline Type & article \\
\hline File Information & A PL95-5_053116.pdf \\
\hline
\end{tabular}

Instructions for use 


\title{
Near-IR vibrational dynamics of periodic gold single and pair nanocuboids
}

\author{
Li Wang, ${ }^{1}$ Yoshiaki Nishijima, ${ }^{2}$ Kosei Ueno, ${ }^{2}$ Hiroaki Misawa, ${ }^{2, a)}$ and Naoto Tamai ${ }^{1, a)}$ \\ ${ }^{1}$ Department of Chemistry, School of Science and Technology, Kwansei Gakuin University, 2-1 Gakuen, \\ Sanda, Hyogo 669-1337, Japan \\ ${ }^{2}$ Research Institute for Electronic Science, Hokkaido University, N21 W10 CRIS Building, \\ Sapporo 001-0021, Japan
}

(Received 28 June 2009; accepted 20 July 2009; published online 7 August 2009)

\begin{abstract}
We examined vibrational dynamics of periodic gold single and pair nanocuboids with $9 \mathrm{~nm}$ separation at $400 \mathrm{~nm}$ excitation by near-IR transient absorption spectroscopy. The similar oscillation periods of coherent phonon vibration ( $77 \pm 1 \mathrm{ps)}$ were observed for both nanocuboids while the damping of the pair was faster than that of the single. From the analysis of bleaching peak shift and finite-difference time-domain calculation, the lattice change in coherent phonon vibration was estimated to be $\pm 1 \%$ for a quasiextensional and $\pm 3 \%$ for an quasibreathing modes of a single nanocuboid. (c) 2009 American Institute of Physics. [DOI: 10.1063/1.3202403]
\end{abstract}

Recently, the surface plasmon resonance (SPR) of metal nanostructures has attracted keen interest due to the potential applications in surface-enhanced Raman scattering, optical biosensing, imaging techniques, etc. ${ }^{1,2}$ SPR of metal nanoparticles is a collective oscillation of the conduction electrons and is affected by the geometry, composition of the particles and the environments. SPR frequency in the assembled nanostructures was recently reported to be related with interparticle separation and regularly influenced by the coupling effects. ${ }^{3-6}$ The gold nanoparticles bounded with DNA were used to measure the distance dependence of the plasmon resonance, as called "plasmon rulers." This universal scaling behavior is also suitable for metal nanostructures of complex geometry studied by the electrodynamic simulations. ${ }^{6}$ To understand more profoundly the near-field coupling between well-confined metal nanostructures fabricated by lithography methods, the ultrafast dynamics of those nanostructures have been examined by transient absorption (TA) spectroscopy. ${ }^{7-9}$ The single gold nanoprism in the assembled array was investigated and found to be strong shape sensitivity of vibrational period. ${ }^{8}$ The periodical gold nanodisks were found to obey the similar scaling behavior of the separation dependence of the coherent phonon vibration frequency with the surface plasmon electronic oscillation frequency due to the near-field coupling. ${ }^{9}$ However, it is not so clear whether the frequency of the coherent phonon vibration obeys the universal scaling behavior similar to SPR frequency or not for different geometry of metal nanostructures from nanodisks, for example, nanocuboids.

In this letter, we examined the coherent phonon vibration of periodic single and pair of gold nanocuboids prepared by the electron-beam lithography by near-IR femtosecond TA spectroscopy. The vibration period of coherent phonon was mainly affected by the shape of the nanostructure itself while the lifetime of oscillation of plasmon resonance was strongly influenced by the near-field coupling.

Two types of the periodic gold nanocuboids, single and pair, were fabricated on quartz slides by electron-beam lithography. ${ }^{10}$ The typical atomic force microscopy (AFM)

\footnotetext{
a) Authors to whom correspondence should be addressed. Electronic addresses: misawa@es.hokudai.ac.jp and tamai@kwansei.ac.jp.
}

images of periodic gold nanocuboids were shown in Fig. 1 with the size of $150 \times 150 \times 15 \mathrm{~nm}^{3}$ for each cuboid. The central distance between two single nanocuboids was about $780 \mathrm{~nm}$, as shown in Fig. 1(a). Two nanocuboids called the pair in Fig. 1(b) were adjacent in the direction of their diagonal lines with the gap of $9 \mathrm{~nm}$ between two vertexes and the central distance of each pairs was about $700 \mathrm{~nm}$. TA spectra were measured by pump-probe method with polarized 400 $\mathrm{nm}$ excitation and near IR probe laser pulses combined with a telescope system. Moreover, the short wavelength of probe laser was cut by a IR85 filter to avoid the photofusion of the gold nanocuboids.

Figure 2(a) illustrates the SPR spectra of the single and the pair with the polarization of $-45^{\circ}$ and $+45^{\circ}$ to the perpendicular, respectively, examined by a probe pulse of whitelight continuum. $+45^{\circ}$ means the direction of the long diagonal line of the pair as one unit. The peaks by fitting a Lorentzian to the spectra were $920 \mathrm{~nm}$ for the single irrespective of the polarization and shifted from 953 (Ref. 11) to $997 \mathrm{~nm}$ for the pair corresponding to the probe polarization of $-45^{\circ}$ and $+45^{\circ}$. The SPR band was influenced by the proximity of the individual particles and ruled by the scaling behavior of the interparticle plasmon coupling. ${ }^{12}$ According to the plasmon ruler function of $\Delta \lambda / \lambda_{0}=k \times \exp ^{-s /(\tau \times D)}$, if the single particle resonance wavelength $\lambda_{0}=920 \mathrm{~nm}$, amplitude $k=0.11$, interparticle separation $s=9 \mathrm{~nm}$, decay constant $\tau=0.22$ and particle dimension $D=150 \mathrm{~nm}$, we can get the shift in the plasmon resonance wavelength $\Delta \lambda=77 \mathrm{~nm}^{6}$
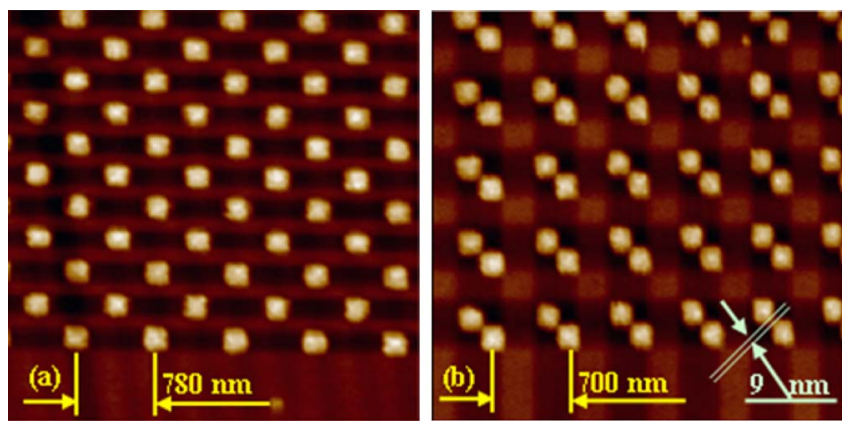

FIG. 1. (Color online) AFM images for the single (a) and pair (b) of gold nanocuboids 

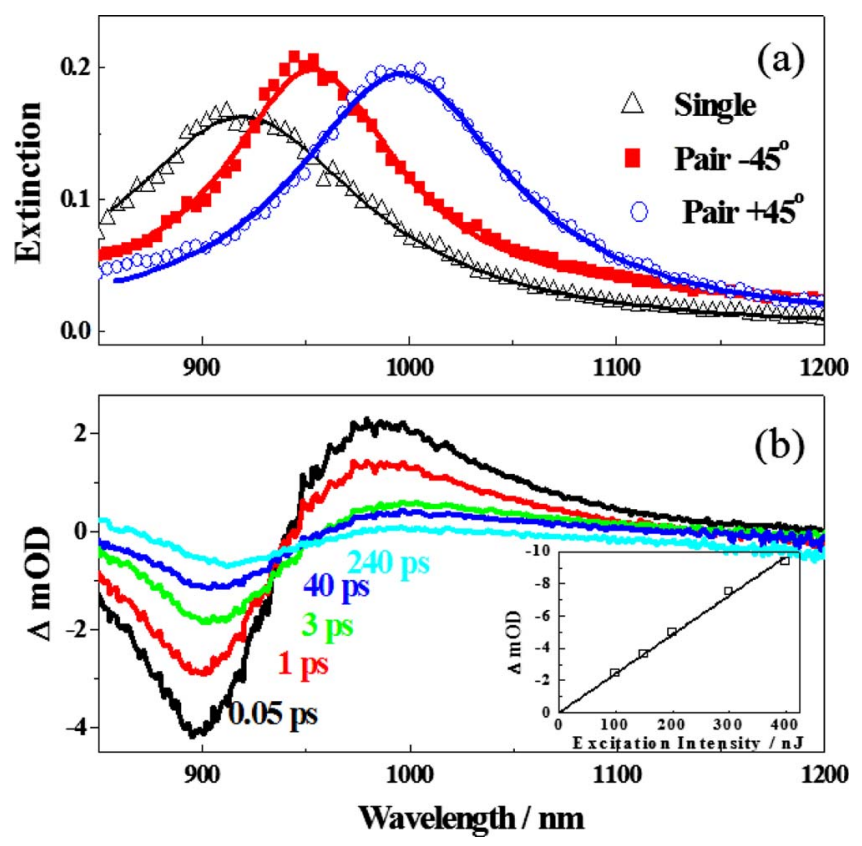

FIG. 2. (Color online) The steady-state extinction spectra of the single and the pair with the polarization of $-45^{\circ}$ and $+45^{\circ}$ to the perpendicular, respectively (a). $+45^{\circ}$ means the direction of the long diagonal line of the pair as one unit. The solid lines were the fitting results by Lorentzian function. TA spectra of the single gold nanocuboids at different times after $400 \mathrm{~nm}$ excitation (b); the inset in (b) is excitation intensity dependence of the minimum $\triangle O D$ of the bleaching peak.

This calculation is in agreement with our experimental result of $997 \mathrm{~nm}(\Delta \lambda=77 \mathrm{~nm})$. Therefore, the redshift of SPR bands was induced by the near-field coupling between the nearest nanocuboids in the arrays.

Figure 2(b) shows TA spectra of the single with the excitation intensity of $200 \mathrm{~nJ}$ at different delay times and the inset is the excitation intensity dependence. The experiment was conducted within the linear absorption region. The TA spectra from 850 to $1130 \mathrm{~nm}$ were represented by a bleaching signal near the SPR band and an absorption signal in the right wing of the band, which is due to the broadening and redshift of the SPR band from the heating of the electrons. ${ }^{13}$

Figure 3(a) illustrates the decay profiles of the single at 880 and $970 \mathrm{~nm}$ corresponding to the two sides of the SPR band. The dynamics of electrons in metal nanoparticles can be described by the electron-electron scattering (a few hundred femtoseconds), electron-phonon coupling (a few picoseconds) and the thermal energy transfer to environments (a few hundred picoseconds). ${ }^{13}$ The slowly damped oscillation following the fast decay were $\sim 180^{\circ}$ out-of-phase for the two curves and similar to the cases of gold nanorods and spherical particles. ${ }^{13-15}$ The decay profiles were fitted by two exponential decays plus a damped cosine function. ${ }^{9}$ The oscillation periods at both wavelengths were 77.3 and $77.7 \mathrm{ps,}$ respectively.

The shift in bleach peaks obtained from the TA spectra were plotted in Fig. 3(b) for single and pair with different polarization. The oscillation similar to absorption dynamics was clearly detected, suggesting that with the impulsive excitation the periodic change of SPR band was induced by the periodic structural change of gold nanocuboid accompanied with the coherent phonon vibration. The peak shift dynamics in Fig. 3(b) were fitted by one exponential decay plus a damped cosine function. The typical fast lifetime was about
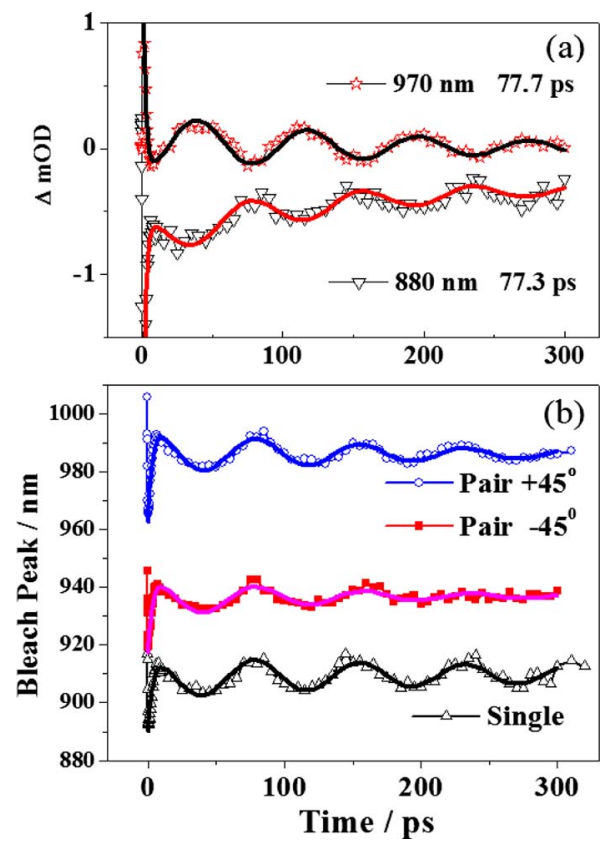

FIG. 3. (Color online) The decay profiles at 880 and $970 \mathrm{~nm}$ with the excitation intensity of $200 \mathrm{~nJ}$ (a); the corresponding shift of the bleaching peaks for the single and pair with two polarization (b). The curves in [(a) and (b)] were fitted by exponential decays plus a damped cosine function.

3 ps which might come from the electron-phonon coupling. The slow lifetime was about 300 and 150 ps for the single and the pair, respectively, which could be contributed by the thermal energy transfer process. Hence the damped oscillation of the single has longer lifetime than that of the pair. Staleva and Hartland ${ }^{16}$ have reported that the lifetime of the vibrational mode was strongly affected by the particle's local environments examined with single particle TA spectroscopy. Thus the different near-field coupling of the single and the pair is probably the main factor of local environmental difference. Moreover, the lifetime of the vibrational mode was also determined by polydispersity of the samples. ${ }^{14}$ From the analysis of the AFM images, the deviation of mean height is $\pm 0.4 \mathrm{~nm}$ for the single while $\pm 1.1 \mathrm{~nm}$ for the pair. This may be another reason for the faster damping of the pair. The 77.0 ps oscillation periods of the single was very similar to those of the pair 76.5 and 78.5 ps with the polarization of $-45^{\circ}$ and $+45^{\circ}(\sim 13 \mathrm{GHz}$ by Fourier transformation). This result suggests that the oscillation period of the pair is not affected by the plasmon coupling even though the gap separation is as short as $9 \mathrm{~nm}$. Moreover, the coherent phonon vibration cannot follow the scaling behavior with respect to the gap, although the detail analyses for pairs with various gap distances should be required.

Two types of vibrational modes may be excited in metal nanostructures by laser-induced heating: breathing mode and extensional mode. The breathing mode, simply radial expansion, was assigned to the metal nanocubes and -prisms assembled on the slides. ${ }^{9,16}$ The extensional modes, an axial expansion combined with a radial contraction, was observed in vibration of metal nanorods. ${ }^{14,17}$ Simplification of two modes was performed on the assumption that either aspect ratio or volume of single nanocuboid can be variable. The diagrams of the two models are shown in Figs. 4(a) and 4(b). Two simplified vibrational modes were used in the simulation by the finite-difference time-domain (FDTD) method to 
(a) Quasi-extensional mode

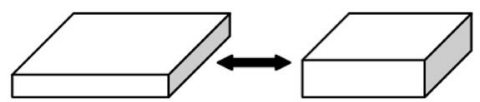

(b) Quasi-breathing mode

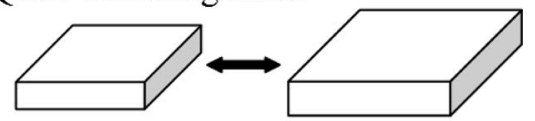

(c)

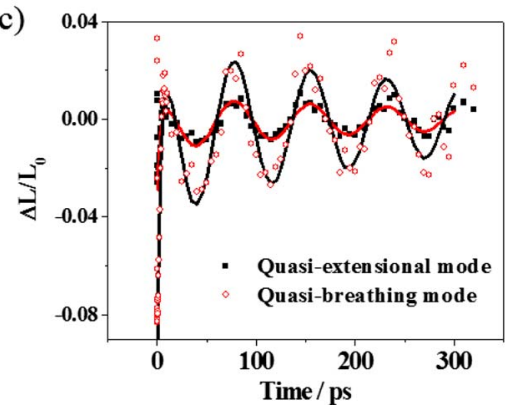

FIG. 4. (Color online) The schematic diagrams of model 1, quasiextensional mode with constant volume and variable aspect ratio (a); and model 2, quasibreathing mode with variable volume and constant aspect ratio (b); side length change $\left(\Delta L / L_{0}\right)$ as a function of time for two modes in the single nanocuboid array calculated by FDTD method (c).

estimate the relative change of the size of the single nanocuboid as shown in Fig. 4(c). Model 1, quasiextensional mode, with constant volume and variable aspect ratio shows the oscillation of one side length is $\sim 1.5 \mathrm{~nm}$, about $\pm 1 \%$ of the original length $150 \mathrm{~nm}$, while model 2, quasibreathing mode, with variable volume and constant aspect ratio presents the oscillation of one side length is $\sim 5 \mathrm{~nm}( \pm 3 \%)$. However, the amplitude of size change is also influenced by the pump intensity, and the coherent phonon vibration for nanometal structures deposited on the slides such as gold nanoprisms, nanodiscs and silver nanocubes was assigned to breathing mode. ${ }^{8,9,16}$ Therefore, we would like to keep the possibilities for quasiextensional and -breathing modes and discuss in the subsequent report.

In conclusion, we investigated the ultrafast dynamics of periodical gold nanocuboids by near IR TA spectroscopy.
The SPR band and the damping lifetime were influenced by the near-field coupling of the nanocuboids while the oscillation period of the coherent phonon was almost the same for the single and the pair. The effect of the interparticle coupling on the plasmon oscillation and the coherent phonon vibration with the small separations is now in progress systematically.

Acknowledgments are made to Grant-in-Aid for Scientific Research on Priority Areas of Strong Photon-Molecule Coupling Fields (No.470) from MEXT, Japan, for support of this research.

${ }^{1}$ S. Link and M. A. El-Sayed, Annu. Rev. Phys. Chem. 54, 331 (2003).

${ }^{2}$ K. A. Willets and R. P. Van Duyne, Annu. Rev. Phys. Chem. 58, 267 (2007).

${ }^{3}$ K.-H. Su, Q.-H. Wei, X. Zhang, J. J. Mock, D. R. Smith, and S. Schultz, Nano Lett. 3, 1087 (2003).

${ }^{4}$ L. Gunnarsson, T. Rindzevicius, J. Prikulis, B. Kasemo, M. Käll, S. Zou, and G. C. Schatz, J. Phys. Chem. B 109, 1079 (2005).

${ }^{5}$ B. M. Reinhard, M. Siu, H. Agarwal, A. P. Alivisatos, and J. Liphardt, Nano Lett. 5, 2246 (2005).

${ }^{6}$ P. K. Jain and M. A. El-Sayed, J. Phys. Chem. C 112, 4954 (2008).

${ }^{7}$ W. Huang, W. Qian, and M. A. El-Sayed, Nano Lett. 4, 1741 (2004).

${ }^{8}$ J. Burgin, P. Langot, N. Del Fatti, F. Vallée, W. Huang, and M. A. ElSayed, J. Phys. Chem. C 112, 11231 (2008).

${ }^{9}$ W. Huang, W. Qian, P. K. Jain, and M. A. El-Sayed, Nano Lett. 7, 3227 (2007).

${ }^{10}$ K. Ueno, V. Mizeikis, S. Juodkazis, K. Sasaki, and H. Misawa, Opt. Lett. 30, 2158 (2005).

${ }^{11}$ If the polarization of the probe is $-45^{\circ}$, the same SPR band of the pair should be excited as the single. But here are 953 and $920 \mathrm{~nm}$. The difference might be due to the short separation between the pairs. If the separation is 2.5 times larger than the pair diameter, the SPR could be the same (Ref. 12). The diameter of pair is about $300 \mathrm{~nm}$. The separation from our AFM results is only $400 \mathrm{~nm}$. Therefore, the redshift of the SPR band in the pair was observed as compared with the single.

${ }^{12}$ P. K. Jain, W. Huang, and M. A. El-Sayed, Nano Lett. 7, 2080 (2007).

${ }^{13}$ G. V. Hartland, Annu. Rev. Phys. Chem. 57, 403 (2006).

${ }^{14}$ M. Hu, X. Wang, G. V. Hartland, P. Mulvaney, J. P. Juste, and J. E. Sader, J. Am. Chem. Soc. 125, 14925 (2003).

${ }^{15}$ G. V. Hartland, J. Chem. Phys. 116, 8048 (2002).

${ }^{16}$ H. Staleva and G. V. Hartland, J. Phys. Chem. C 112, 7535 (2008).

${ }^{17}$ P. Zijlstra, A. L. Tchebotareva, J. W. M. Chon, M. Gu, and M. Orrit, Nano Lett. 8, 3493 (2008). 